\title{
Transcatheter Intervention for Non-variceal Gastrointestinal Bleeding: What Have We Learned in 45 Years?
}

\author{
Doumit S. BouHaidar • Brian J. Strife
}

Published online: 4 June 2013

(c) Springer Science+Business Media New York 2013

Despite considerable advances in diagnostic testing, medical treatment, surgical and non-surgical interventions, therapy of gastrointestinal (GI) bleeding remains a challenge for physicians. Although GI bleeding ceases spontaneously in roughly $75 \%$ of patients, it still carries a relatively high morbidity and mortality in our aging population [1]. When faced with a bleeding patient, clinicians initially attempt to separate hemorrhage arising in the upper gastrointestinal (UGI) tract from that originating in the lower gastrointestinal (LGI) tract, delineated by the ligament of Treitz, due to the considerable differences in diagnosis, treatment and prognosis associated with each region. Regardless of location, endovascular interventions remain important for the diagnosis and treatment of GI bleeding, likely only increasing in application and efficacy in the coming decades.

Before 1968, endovascular physicians had little to offer the acutely bleeding patient. The first major therapeutic intervention for GI bleeding was catheter directed intraarterial vasopressin infusion [2]. A somewhat cumbersome procedure that has largely been abandoned in contemporary practice, vasoconstrictor infusion offered a minimally invasive therapy that could be employed in stable and unstable patients alike, for variceal and non-variceal hemorrhage [3]. In the 1970s, arterial embolization initially

\footnotetext{
D. S. BouHaidar $(\square)$

Department of Internal Medicine, Interventional Endoscopy, Virginia Commonwealth University Health Center, Box 980341, Richmond, VA 23298-0341, USA

e-mail: dsbouhaidar@vcu.edu

\section{B. J. Strife}

Vascular and Interventional Radiology, Virginia Commonwealth University Health Center, Richmond, VA 23298, USA

e-mail: strifebj@vcu.edu
}

appeared promising, although its widespread adoption was thwarted by unacceptably high complication rates, primarily colonic ischemia [4]. When technological advances in imaging, embolic agents, and catheters caught up with physician ingenuity in the late 1990s and early 2000s, a paradigm switch occurred with arterial embolization assuming primacy for catheter-based therapy of UGI and LGI bleeding.

In the past several years, numerous studies, predominantly retrospective series, have reported the safety and efficacy of arterial embolization for UGI and LGI bleeding [5-7]. Although published algorithms for the management of GI bleeding exist, the application of mesenteric angiography with transcatheter arterial embolization in the therapy of GI hemorrhage remains controversial.

The study by Yap et al. [8] in this issue describes a single-center experience with transcatheter arterial embolization for non-variceal GI bleeding over a period of 8 years. The authors demonstrate a high technical success rate in keeping with other reported series and national guidelines. Of note, the data are reported without strict separation of UGI from LGI bleeding, which dilutes their conclusions. Despite this limitation, the authors provide a detailed look at patients requiring transcatheter arterial embolization, and identify important risk factors for rebleeding, notably underlying peptic ulcer disease and coagulopathy.

Their experience highlights an important concept in the endovascular treatment for GI bleeding; embolization in the UGI tract can either be "targeted" at visualized arterial abnormalities, or "empiric", where the suspected culprit arterial branch is occluded despite no demonstrated angiographic abnormality. In the LGI tract, where the collateral arterial supply is tenuous, targeted embolization is recommended such as in the accompanying article, 
where targeted embolization was used in all LGI bleeds. In the UGI tract, rebleeding occurred more frequently in targeted $(29 \%)$ compared with empiric $(23 \%)$ embolization, yet the 30-day mortality was 11 and $31 \%$, respectively. With no clear explanation for the disparity, additional investigation is warranted with a goal for improved outcomes in patients relegated to empiric embolization in the UGI tract.

Although the study focuses on embolization, 117/212 patients underwent diagnostic mesenteric angiography without embolization. Hemorrhagic-specific mortality at 30 days was $19 \%$ in the no embolization group, compared with $14 \%$ among empiric and $7 \%$ among targeted embolizations. While a generalized conclusion can be made that embolization reduced hemorrhage-specific mortality, a detailed description of patient characteristics in the treatment and non-treatment groups was not provided. By focusing on those patients who did not undergo embolization, it may be possible to identify weaknesses in diagnostic and interventional techniques.

The authors noted that endoscopy helped localize the site of GI bleeding. For UGI bleeding, endoscopy localized the source in $81 \%(56 / 69)$, ultimately guiding the selection of the arterial branch for embolization in $86 \%(31 / 36)$. LGI bleeding was less impacted by endoscopy, with only $24 \%(4 / 17)$ of bleeding sources localized. Tagged red blood cell (RBC) scanning, a sensitive nuclear medicine test for active bleeding, provided LGI bleed localization in an impressive $92 \%(12 / 13)$. As noted by the authors, this difference primarily impacts transcatheter arterial embolizations in the UGI tract where an empiric or targeted technique can be used. It is not clear how endoscopy and tagged RBC scanning impact embolizations in the LGI tract, where empiric technique is not customarily applied.

When GI bleeding is suspected, appropriate resuscitation, medical therapy, and correction of coagulopathy should ensue. In suspected UGI bleeding, endoscopy should be the primary study for diagnosis and potential treatment. When bleeding is refractory to endoscopic treatment or recurs after treatment, mesenteric angiography with targeted or empiric embolization can be pursued with high technical success, good efficacy, and low complication rate, as demonstrated in the current study and by others [6-8]. For those patients who fail these initial measures, repeat procedures and/or surgery may be necessary. In LGI bleeding, similar guidelines apply, although the initial best test is not as evident. Options include lower endoscopy, tagged RBC scanning, and diagnostic mesenteric angiography. Depending on the results, therapeutic interventions can be pursued, similar to UGI bleeding.

Significant challenges remain in the management of GI bleeding. An under-discussed subject is the large number of patients, such as the $55 \%$ in the accompanying study, for whom angiography is only diagnostic. More specifically, LGI bleeding evaluated with mesenteric angiography is overwhelmingly diagnostic [9]. Since angiography is resource intensive and invasive, additional tools and techniques are needed to increase the percentage of positive examinations.

Two emerging diagnostic techniques with promising results are computed tomography angiography (CTA) and provocative mesenteric angiography (PMA). CTA evaluation of GI bleeding using a multiphase intravenous contrasted enhanced technique accurately identified the source of hemorrhage with high sensitivity $(85 \%)$ and specificity (92\%) in a large meta-analysis [10]. In a recent series, 86 patients with acute GI bleeding were studied with CTA, yielding 22 positive and 64 negative examinations. Among the patients with a positive CTA, 17 underwent angiography with targeted embolization performed in $71 \%$. Three others went directly to curative surgery with no intervening angiogram. Importantly, $92 \%$ of patients with a negative CTA underwent medical therapy requiring no further intervention [11]. CTA has distinct advantages compared with tagged RBC scanning and diagnostic angiography: (1) CT scanners are readily available in the US, (2) a CTA study can be performed rapidly with no preparation necessary other than the required intravenous access, (3) published protocols specifically geared towards detection of GI bleeding are readily available, (4) a positive study, classically defined as intraluminal extravasation of iodinated contrast, provides precise anatomic localization of the bleeding and often the underlying cause (e.g., diverticulum or tumor), and (5) additional information can be gleaned from the study, such as an unrecognized bowel perforation, which may direct a patient to surgery rather than angiography or endoscopy. Tagged RBC scanning requires preparation of the radionuclide, longer imaging time, and can provide only approximate localization. While its sensitivity for detecting bleeding at rates less $\leq 0.1 \mathrm{ml} / \mathrm{min}$ is well known, CTA compares favorably detecting bleeding as slow as $0.3 \mathrm{ml} / \mathrm{min}$. Angiography is an invasive test, requires significant resources that may not be immediately available, and has the least sensitivity for detection of bleeding $(>0.5 \mathrm{ml} / \mathrm{min})$. At our institution, CTA has become the preferred initial radiologic examination for acute GI bleeding not localized by upper endoscopy.

Once a patient is considered for mesenteric angiography, identifying the bleeding source can be challenging due to the low rate of positive studies, particularly in LGI bleeding where direct visualization is necessary for embolization. In 2001, physicians at Duke University Medical Center published their initial experience with PMA using a combination of intravenous heparin, intraarterial tissue plasminogen activator, and intra-arterial tolazoline in patients with negative angiography for LGI 
bleeding. Using their protocol, a previously occult bleeding source was identified in six of 17 procedures $(35 \%)$ with no complications encountered. Successful treatment was achieved in five of six patients [12]. In 2010, the same group published their subsequent experience with PMA using a modified protocol. They elicited angiographically visible bleeding in 11 of 36 procedures $(31 \%)$, resulting in ten successful embolizations with no hemorrhagic complications [9]. As noted by Kim et al. [9], the published literature on PMA is limited at this time, but their experience and that of others indicates that PMA is safe and effective, and should undergo additional investigation on a larger scale.

In conclusion, the landscape for transcatheter arterial embolization in GI bleeding continues to change, with published reports from authors like Yap et al. offering valuable insight into the application of the technique and where future investigation is warranted. Emerging diagnostic techniques like CTA and PMA present opportunities to dramatically improve patient selection and outcomes for transcatheter arterial embolization.

Conflict of interest None.

\section{References}

1. van Leerdam ME. Epidemiology of acute upper gastrointestinal bleeding. Best Pract Res Clin Gastroenterol. 2008;22:209-224.

2. Nusbaum M, Baum S, Kuroda K, Blakemore WS. Control of portal hypertension by selective mesenteric arterial drug infusion. Arch Surg. 1968;97:1005-1014.
3. Rosch J, Dotter CT, Antonovic R. Selective vasoconstrictor infusion in the management of arterio-capillary gastrointestinal hemorrhage. AJR Radium Ther Nucl Med. 1972;116:279-288.

4. Darcy M. Treatment of lower gastrointestinal bleeding: vasopressin infusion versus embolization. J Vasc Interv Radiol. 2003; 14:535-543.

5. Kuo WT, Lee DE, Saad W, Patel N, Sahler LG, Waldman DL. Superselective microcoil embolization for the treatment of lower gastrointestinal hemorrhage. J Vasc Interv Radiol. 2003;14: 1503-1509.

6. Padia SA, Geisinger MA, Newman JS, Pierce G, Obuchowski NA, Sands MJ. Effectiveness of coil embolization in angiographically detectable versus non-detectable sources of upper gastrointestinal hemorrhage. J Vasc Interv Radiol. 2009;20: 461-466.

7. Schenker MP, Duszak R, Soulen MC, et al. Upper gastrointestinal hemorrhage and transcatheter embolotherapy: clinical and technical factors impacting success and survival. J Vasc Interv Radiol. 2001;12:1263-1271.

8. Yap FY, Omene BO, Patel MN, et al. Transcatheter embolotherapy for gastrointestinal bleeding: a single center review of safety, efficacy, and clinical outcomes. Dig Dis Sci. (Epub ahead of print). doi:10.1007/s10620-012-2547-z.

9. Kim CY, Suhocki PV, Miller MJ, Khan M, Janus G, Smith TP. Provocative mesenteric angiography for lower gastrointestinal hemorrage: results from a single-institution study. J Vasc Interv Radiol. 2010;21:477-483.

10. García-Blázquez V, Vicente-Bártulos A, Olavarria-Delgado A, et al. Accuracy of CT angiography in the diagnosis of acute gastrointestinal bleeding: systematic review and meta-analysis. Eur Radiol. 2013;23:1181-1190. doi:10.1007/s00330-012-2721-x.

11. Kennedy DW, Laing CJ, Tseng LH, Rosenblum DI, Tamarkin SW. Detection of active gastrointestinal hemorrhage with CT angiography: a 4.5 year retrospective review. J Vasc Interv Radiol. 2010;21:848-855.

12. Ryan JM, Key SM, Dumbleton SA, Smith TP. Nonlocalized lower gastrointestinal bleeding: provocative bleeding studies with intraarterial TPA, heparin, and tolazoline. J Vasc Interv Radiol. 2001;12:1273-1277. 